\title{
A Unified Approach to Performance Evaluation of Switched Diversity in Independent and Correlated Fading Channels ${ }^{*}$
}

\author{
A. Annamalai ${ }^{1}$, C. Tellambura ${ }^{2}$ and V. K. Bhargava ${ }^{1}$ \\ 1. Department of Electrical and Computer Engineering, University of Victoria, PO Box 3055 STN CSC, Victoria \\ BC V8W 3P6, Canada, Tel: +1-250-721-6043, Fax: +1-250-721-6048, E-mail: bhargava@ece.uvic.ca
}

2. School of Computer Science and Software Engineering, Monash University, Clayton, Victoria 3168, Australia Tel: +1-613-9905-3296, Fax: +1-613-9905-3402, E-mail: chintha@dgs.monash.edu.au

\begin{abstract}
This paper outlines a unified approach to performance evaluation of a broad class of coherent, differentially coherent and noncoherent digital communication systems with dual-branch switched diversity (SWC) reception over generalized fading channels. The moment generating function (MGF) of the signal power at the output of the SWC combiner and the first-order derivative of the MGF with respect to the switching threshold are derived. These expressions are obtained for the general case of correlated fading and nonidentical diversity branches, and hold for any common fading distributions (e.g., Rayleigh, Nakagami-m, Rician, Nakagami-q). The optimum switching threshold (in a minimum error rate sense) is obtained by solving a nonlinear equation which is formed by using the first-order derivative of the MGF. This nonlinear equation can be simplified for several special cases: (a) closed-form expressions for the optimal switching threshold are derived for three generic forms of the conditional error probability by assuming independent and identically distributed diversity branches; (b) a closed-form formula for the optimal switching threshold is derived for the non-coherent binary modulation formats in correlated Rayleigh or Nakagami-m fading with identical fading statistics.
\end{abstract}

\section{INTRODUCTION}

The ideal selective combining (SDC) that selects the branch with the highest signal-to-noise ratio (SNR) may not be practical for radio links that use continuous transmission (e.g., FDMA systems) because it requires continuous monitoring of all the diversity branches. This problem can be circumvented by adopting a suboptimal switched diversity scheme.

Previous related studies on the switched diversity systems include the following: In [1], Rustako et. al. theoretically and experimentally examined a switched diversity system on independent Rayleigh channels using a continuous time signal model. In their switch-and-stay strategy, switching between the two antennas only occurs if there is a downward threshold crossing. Shortall [2] builds a prototype of a switched diversity system and experimentally examine the effect of the signal correlation on the receiver performance. Subsequently, Adachi et. al. [3] investigated the performance of a periodic switching diversity technique using digital FM with discriminator detection on Rayleigh fading channels. Blanco and

\footnotetext{
* This work was supported in part by a Strategic Project Grant from the Natural Sciences and Engineering Research Council (NSERC) of Canada and in part by Telus Mobility.
}

Zdunek [4] theoretically examined the performance of switched diversity system initially proposed in [1] for noncoherent frequency shift keying (NCFSK) in independent Rayleigh fading channels based on a discrete-time approach. Their analysis was extended in [5] for the Nakagami-m fading channels. In [6], Abu-Dayya and Beaulieu proposed a different switch-and-stay strategy (which is referred to as SWC in this paper) and analyzed the performance of binary NCFSK on Nakagami-m fading channel based on a discrete-time model. Different from [4], the antenna switch in the SWC scheme is activated in the next switching instant as long as the measured local power in the current antenna is below the threshold level (i.e., the envelope of the received signal need not necessarily cross the threshold in the negative direction). Therefore, it does not require comparison of present samples with past samples. Moreover, the rate of branch switching is reduced with respect to the ideal selection diversity, which translates into a reduction of transient effects due to switching. Their analysis for both independent and correlated Nakagami-m signal fading has been extended to Rician fading in [7]. Numerical results for BPSK, 8-PSK and 16-QAM with independent and identically distributed dual-branch SWC in Nakagami-m fading can be found in [8]. The authors' also derived the optimum switching threshold for BPSK in a closed-form in Rayleigh and Nakagami-m fading channels.

By contrast, here we derive a generic formula to study the performance of SWC for a broad class of binary and M-ary modulation formats in a myriad of fading environments. Different from [6], [7] and [8], we directly determine the MGF of the resultant signal power statistic without imposing any restrictions. In fact, the signal statistics from different diversity branches may even be modelled using different families of distribution (i.e., mixed-fading). In particular, we examine the effect of power imbalance on the diversity receiver performance and the optimal switching threshold. This is an important consideration because in practice identical fading statistics across the diversity branches are rarely available. However, all the previous theoretical studies only considered the case of identical diversity branches for analytical simplicity. Once the MGF is available, we can express the average symbol error rate (ASER) in terms of a finite-range integral involving only the MGF. Since the derivative of the MGF with respect to the switching threshold can be obtained at once, the optimum switching threshold can be readily expressed in a 
closed-form in many instances.

\section{STATISTICAL CHARACTERIZATION OF THE SNR AT THE OUTPUT OF THE SWC COMBINER}

Table 1 in [9] summarizes the instantaneous SER for a wide range of modulation schemes in an AWGN channel. Recognizing the alternative exponential form for the complementary error functions, i.e., $\operatorname{erfc}(\sqrt{\gamma})=\frac{2}{\pi} \int_{0}^{\pi / 2} \exp \left(-\gamma \csc ^{2} \theta\right) d \theta$ and $\operatorname{erfc}^{2}(\sqrt{\gamma})=\frac{4}{\pi} \int_{0}^{\pi / 4} \exp \left(-\gamma \csc ^{2} \theta\right) d \theta$, we can express these conditional error probabilities (for binary and M-ary signal constellations) as a special case of the following generic form,

$$
P_{S}(\varepsilon \mid \gamma)=\sum_{k} \int_{0}^{n_{k}} a_{k}(\theta) \exp \left(-\gamma b_{k}(\theta)\right) d \theta
$$

where $a_{k}(\theta)$ and $b_{k}(\theta)$ are coefficients independent of $\gamma$ but may be dependent on $\theta$.

The ASER in the fading channels with switched diversity can be derived by averaging the conditional error probability over the probability density function (PDF) of SNR at the output of the switched combiner in a specified fading environment. It is more insightful if we employ the MGF approach [10]-[12] since the ASER can be expressed in terms of only the MGF of the resultant SNR. Further, closed-form formulas for the computing the optimum switching threshold can be determined in a straight-forward fashion for all common fading channels and for different modulation schemes if the diversity branches have identical fading statistics. Hence, in the following we will derive the MGF of the local power at the output of the switched combiner for both correlated and independent signal fading cases.

\section{A. Correlated Fading and Nonidentical Diversity Branches}

Similar to [4]-[7], our analysis is based on a discrete time model. The switching is performed at discrete instants of time $t=n T$, where $n$ is an integer, and $T$ is the interval between switching instants. The cumulative distribution function (CDF) of the resultant signal power at the output of the SWC combiner can be written as [6, Eq. (4)],

$$
\begin{aligned}
F_{z_{n}}(u) & =\operatorname{Pr}\left\{z_{n} \leq u\right\} \\
& =\operatorname{Pr}\left\{z_{n}=x_{n} \text { and } x_{n} \leq u\right\}+\operatorname{Pr}\left\{z_{n i}=y_{n} \text { and } y_{n} \leq u\right\}
\end{aligned}
$$

where $x_{n}$ and $y_{n}$ denotes the local powers of the signals received by the two antennas at $t=n T$, and $z_{n}$ is the local signal power at the output of the switched diversity receiver at $t=n T$ (see Fig. 1).

The corresponding MGF of $z_{i n}$ has been derived in [14],

$$
\begin{gathered}
\phi_{\Sigma}(s)=\left[\int_{\xi}^{x} \exp (-s u) f_{i}(u) d u+\int_{0}^{k} \phi_{y}(s, X) d X\right] \operatorname{Pr}\{z=x\} \\
+\left[\int_{\xi}^{x} \exp (-s u) f_{y}(u) d u+\int_{0}^{\vdots} \phi_{x}(s, Y) d Y\right] \operatorname{Pr}\{z=y\}
\end{gathered}
$$

where the parameters $\phi_{x}(s, Y)=\int_{0}^{x} f_{x, y}(u, Y) \exp (-s u) d u$ and $\phi_{y}(s, X)=\int_{0}^{x} f_{s, y}(X, u) \exp (-s u) d u$ are the marginal MGFs. Notations $f_{x}($.$) and F_{x}($.$) correspond to the PDF and CDF of$ the signal power for antenna $x$, respectively; and $f_{x, y}(.,$.$) is$ the joint PDF of $x_{n}$ and $y_{n}$.

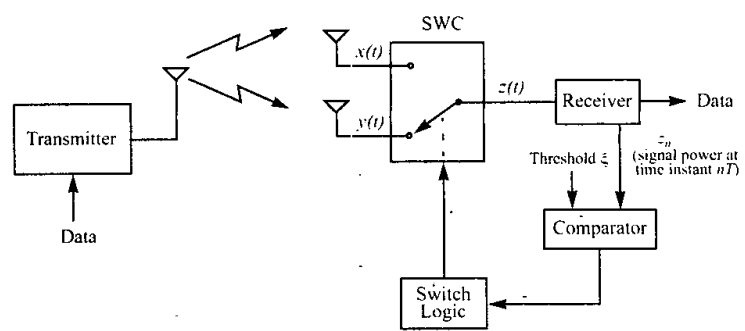

Fig 1. Block diagram of a predetection switched diversity system.

Now let us calculate the antenna selection probabilities $\operatorname{Pr}\{z=x\}$ and $\operatorname{Pr}\{z=y\}$. If both diversity branches have identical fading statistics, then each of the two antennas will have an equal chance of being selected. However, when the sequences $\left\{x_{n}\right\}$ and $\left\{y_{n}\right\}$ are not identically distributed due to power imbalance, then the likelihood of staying in a "good" diversity branch will be higher because a branch with a higher mean received signal power will be favored most of the time. The branch selection probabilities may be computed using a two-state Markov chain shown in Fig. 2.

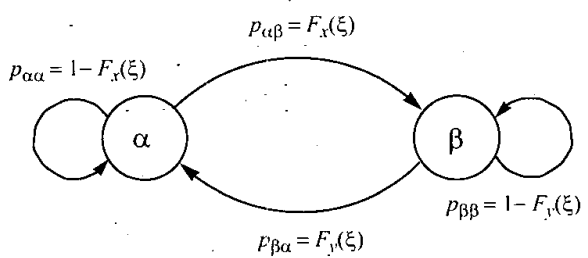

Fig 2. A two-state Markov chain for calculating the antenna selection probabilities.

The states $\alpha$ and $\beta$ correspond to the event that antenna $x$ and antenna $y$ is selected, respectively. The state transition probabilities, $p_{i j}$, is dictated by the probability that the power on a specified branch is either greater or smaller than the preset threshold. The steady-state solution to this Markov chain yields the antenna selection probabilities:

$$
\begin{gathered}
\operatorname{Pr}\{z=x\}=\frac{p_{\beta a}}{p_{\alpha \beta}+p_{\beta \alpha}}=\frac{F_{y}(\xi)}{F_{x}(\xi)+F_{y}(\xi)} \equiv A(\xi) \\
\operatorname{Pr}\{z=y\}=\frac{p_{\alpha \beta}}{p_{\alpha \beta}+p_{\beta \alpha}}=\frac{F_{x}(\xi)}{F_{x}(\xi)+F_{y}(\xi)} \equiv 1-A(\xi)
\end{gathered}
$$

Since $\int_{x}^{\infty} \exp (-s u) f_{x}(u) d u=\phi_{.}(s)-\int_{0}^{5} \exp (-s u) f_{x}(u) d u$ and substituting (4) and (5) into (3), we obtain a general expression for the MGF of the local signal power at the output of the switched combiner taking into account of the branch correlation as well as the dissimilar fading statistics, i.e.,

$$
\begin{aligned}
& \phi_{z}(s)=A(\xi)\left\{\phi_{x}(s)+\int_{0}^{\xi}\left[\phi_{y}(s, X)-\exp (-s X) f_{x}(X)\right] d X\right\} \\
& +[1-A(\xi)]\left\{\phi_{y}(s)+\int_{0}^{\xi}\left[\phi_{x}(s, Y)-\exp (-s Y) f_{y}(Y)\right] d Y\right\}
\end{aligned}
$$

where $\phi_{x}($.$) and \phi_{y}($.$) are the MGF of the signal power in$ antenna $x$ and $y$, respectively (see Table 1 for a list of MGF of the signal power for several common fading channels). To the best of our knowledge, this result is new. From (1), the ABER or-ASER with SWC is simply

$$
P_{S}=\sum_{k} \int_{0}^{n_{k}} a_{k}(\theta) \phi_{i}\left(b_{k}(\theta)\right) d \theta
$$




\section{B. Independent Fading and Nonidentical Diversity Branches}

If the antenna separation is greater than half-wavelength then it is reasonable to assume that the two diversity branches will be independent. However, their signal strength and fading severity index may be different in an actual mobile link since the radio waves take different propagation paths and may undergo different fading before arriving at the receiver. Recognizing $\phi_{y}(s, X)=f_{x}(X) \phi_{y}(s)$ and $\phi_{x}(s, Y)=f_{y}(Y) \phi_{x}(s)$ when the two diversity branches are statistically independent, the MGF of $z_{n}$ shown in (6) reduces to

$$
\begin{aligned}
& \phi_{z}(s)=\dot{A}(\xi)\left\{\int_{\xi}^{x} \exp (-s u) f_{x}(u) d u+\phi_{y}(s) F_{x}(\xi)\right\} \\
& +[1-A(\xi)]\left\{\phi_{s}(s) F_{y}(\xi)+\int_{\xi}^{x} \exp (-s u) f_{y}(u) d u\right\}
\end{aligned}
$$

Notice that (8) is still valid even if the received signal envelope in different antennas are modelled from different families of the fading distribution (i.e., mixed-fading model). As before, the ASER of the SWC with uncorrelated diversity branches is given by (7). However, $\phi_{-}($.$) is evaluated using$ (8) instead of (6).

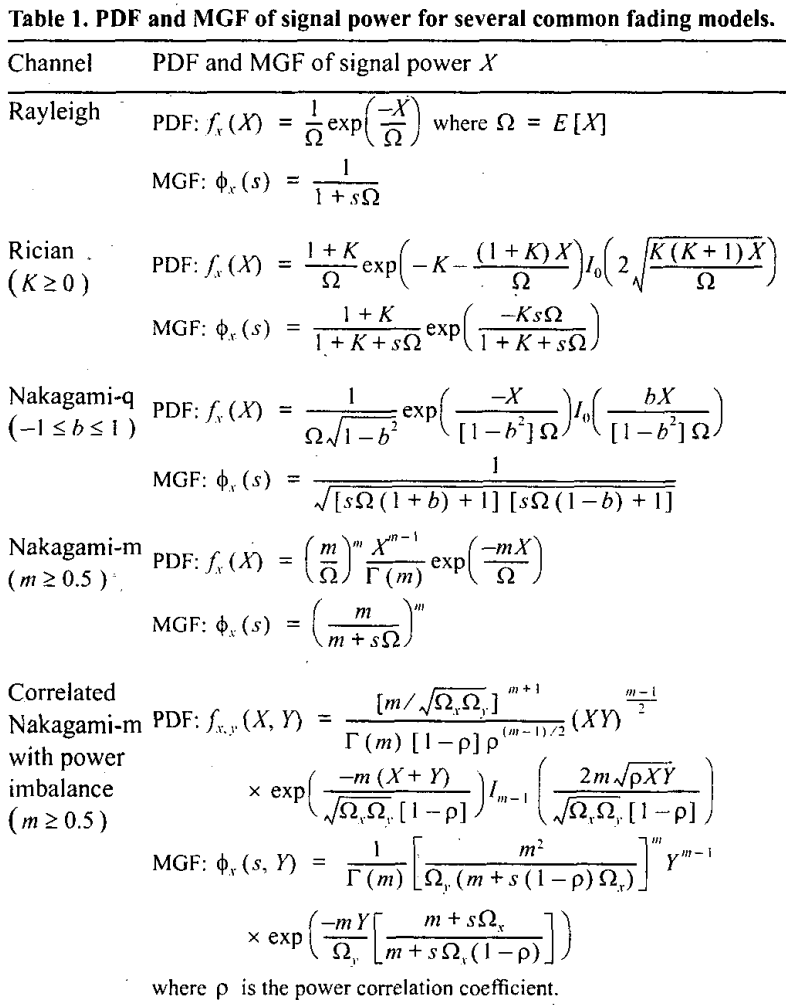

\section{Correlated Fading and Identical Diversity Branches}

If the samples $x_{n}$ and $y_{n}$ are identically distributed, then $\operatorname{Pr}\{z=x\}=\operatorname{Pr}\{z=y\}=1 / 2, \quad \phi_{x}(s,)=.\phi_{y}(s,$.$) \quad and$ $F_{x}()=.F_{r}($.$) . Owing to the symmetry, the MGF of z_{n}$ illustrated in (6) may now be simplified as

$$
\phi_{s}(s)=\phi_{x}(s)+\int_{0}^{\xi}\left[\phi_{x}(s, Y)-\exp (-s Y) f_{y}(Y)\right] d Y
$$

\section{Independent Fading and Identical Diversity Branches}

Following our treatment in Section IIC, it is straight-forward to show that $(8)$ reduces to $(10)$ for the independent and identically distributed diversity branches:

$$
\phi_{z}(s)=\phi_{x}(s) F_{x}(\xi)+\int_{\xi}^{\infty} \exp (-s u) f_{x}(u) d u
$$

\section{OPTIMIZATION OF THE SWC STRATEGY}

If the switching threshold $\xi$ is set to be very large, then there will be constant switching between the two antennas because the probability of the received signal exceeding $\xi$ will be small. In this case, the performance of SWC will be equivalent to the performance of a diversity branch selected in random, which resembles the behavior for no diversity case. On the other extreme (i.e., the value of $\xi$ is set to be very small), the SWC combiner will be stuck in one of the diversity branches because the likelihood of received signal power staying above the specified threshold increases. Once again, the performance of the SWC will be close to the single diversity branch case. It is evident that the performance of switched diversity strategy is dependent on the selection of the switching threshold, and proper choice of $\xi$ will minimize the average error probability. Hence in this section, we will derive analytical expressions that will allow us to compute the optimum switching threshold (in the minimum error rate sense) either in a closed-form (for identical fading statistics) or numerically (for nonidentical fading statistics) for a broad class for a broad class of digital modulation formats in arbitrary fading environments.

\section{A. Correlated Fading and Nonidentical Diversity Branches}

Differentiating (6) with respect to $\xi$, we obtain

$$
\begin{gathered}
\frac{\partial}{\partial \xi} \phi_{z}(s)=A(\xi)\left[\phi_{y}(s, \xi)-\exp (-s \xi) f_{x}(\xi)\right] \\
+[1-A(\xi)]\left[\phi_{x}(s, \xi)-\exp (-s \xi) f_{y}(\xi)\right] \\
+B(\xi)\left[\phi_{x}(s)-\phi_{y}(s)+\int_{0}^{\xi}\left\{\phi_{y}(s, u)-\phi_{x}(s, u)\right\} d u\right] \\
+B(\xi) \int_{0}^{\xi}\left[f_{y}(u)-f_{x}(u)\right] \exp (-s u) d u
\end{gathered}
$$

where $A(\xi)$ is defined in (4) and

$$
B(\xi)=\frac{\partial}{\partial \xi} A(\xi)=\frac{F_{x}(\xi) f_{y}(\xi)-F_{y}(\xi) f_{x}(\xi)}{\left[F_{x}(\xi)+F_{y}(\xi)\right]^{2}}
$$

Now our task is to find the optimum $\xi^{*}$ that minimizes the ASER. This value can be determined by solving $\frac{\partial P_{s}}{\partial \xi}=0$ for $\xi$. By differentiating (7) under the integral sign, and exploiting the results of (11), we get

$$
\begin{aligned}
& \frac{d P_{s}}{d \xi}=\sum_{k} \int_{0}^{\eta_{k}}\left[A(\xi) \phi_{y}\left(b_{k}(\theta), \xi\right)+\{1-A(\xi)\} \phi_{x}\left(b_{k}(\theta), \xi\right)\right] \\
& \quad \times a_{k}(\theta) d \theta-P_{S}(\varepsilon \mid \xi)\left[A(\xi) f_{x}(\xi)+\{1-A(\xi)\} f_{y}(\xi)\right] \\
& +B(\xi)\left[P_{s}^{(x)}-P_{s}^{(y)}+\sum_{k} \int_{0}^{\eta_{k}} a_{k}(\theta) \int_{0}^{\xi}\left\{\phi_{y}(s, u)-\phi_{x}(s, u)\right\} d u d \theta\right] \\
& \quad+B(\xi) \sum_{k} \int_{0}^{\eta_{k}} a_{k}(\theta) \int_{0}^{\xi}\left\{f_{y^{\prime}}(u)-f_{x}(u)\right\} \exp (-s u) d u d \theta
\end{aligned}
$$

where notation $P_{S}^{(x)}$ and $P_{S}^{(y)}$ correspond to the ASER of the diversity branch $x$ (i.e., obtained by replacing $\phi_{-}(s)$ in (7) with $\left.\phi_{x}(s)\right)$ and $y$, respectively. For this general case, no 
closed-form solution for $\xi^{*}$ exists. Hence, this value will be determined numerically.

\section{B. Independent Fading and Nonidentical Diversity Branches}

If the diversity branches are assumed to be statistically independent, then we may replace the marginal MGFs in (13) with $\phi_{y}(s, u)=f_{x}(u) \phi_{y}(s)$ and $\phi_{x}(s, u)=f_{y}(u) \phi_{x}(s)$. Then it can be easily shown that the optimal threshold is obtained by solving the following expression:

$$
\begin{aligned}
& A(\xi) f_{x}(\xi) P_{S}^{(y)}+\{1-A(\xi)\} f_{y}(\xi) P_{S}^{(x)}+B(\xi)\left[F_{x}(\xi) P_{S}^{(y)}\right. \\
& \left.-F_{y}(\xi) P_{S}^{(x)}+\sum_{k} \int_{0}^{n_{k}} a_{k}(\theta) \int_{\xi}^{\infty}\left\{f_{y}(u)-f_{x}(u)\right\} \exp (-s u) d u d \theta\right] \\
& \quad-P_{S}(\varepsilon \mid \xi)\left[A(\xi) f_{x}(\xi)+\{1-A(\xi)\} f_{y}(\xi)\right]=0
\end{aligned}
$$

\section{Independent Fading and Identical Diversity Branches}

If both the diversity branches are assumed to be independent and identically distributed, then the PDF, CDF and MGF of $x_{n}$ and $y_{n}$ are interchangeable. Moreover, $A(\xi)=1 / 2$ and $B(\xi)=0$. In this case, (14) reduces to

$$
P_{S}(\varepsilon \mid \xi)=P_{S}^{(x)}=P_{S}^{(v)}
$$

where $P_{s}^{(x)}$ or $P_{S}^{(y)}$ corresponds to the ASER without diversity reception. Next we will identify three special cases of the conditional error probability $P_{S}(\varepsilon \mid \gamma)$ which lend themselves to closed-form formulas for the calculation of $\xi^{*}$ in all common fading environments.

\section{C.1 Exponential Form: $P_{S}(\varepsilon \mid \gamma)=a \exp (-b \gamma)$}

The instantaneous BER of some noncoherent binary modulation schemes (e.g., DPSK and NCFSK) can be expressed in the exponential form. Then $P_{S}=a \phi_{z}(b)$ for the switched diversity receiver, and their corresponding optimum switching threshold is (directly from (15)),

$$
\xi^{*}=\frac{-1}{b} \ln \left(\frac{P_{s}^{(x)}}{a}\right)=\frac{-\ln \left(\phi_{x}(b)\right)}{b}
$$

Substituting $b=1 / 2$ and using appropriate expression for $\phi_{x}($.$) , we arrive to the previous expressions for the optimum$ threshold of NCFSK in Nakagami-m [6, Eq. (14)] and Rician [7, Eq. (12)] fading channels, respectively.

\section{C.2 $P_{s}(\varepsilon \mid \gamma)=a \operatorname{erfc}(\sqrt{b \gamma})$}

The ASER of some coherent binary modulation schemes (e.g., CPSK and CFSK) with SWC is given by $P_{S}=\frac{2 a}{\pi} \int_{0}^{\pi / 2} \phi_{\Sigma}\left(b \csc ^{2} \theta\right) d \theta$. Using (15), we get

$$
\xi^{*}=\frac{1}{b}\left(\operatorname{erfcinv}\left[P_{s}^{(x)} / a\right]\right)^{2}
$$

where erfcinv (.) denotes the inverse of the complementary error function.

\section{C. $3 P_{S}(\varepsilon \mid \gamma)=a \operatorname{erfc}(\sqrt{b \gamma})-c \operatorname{erfc}^{2}(\sqrt{b \gamma})$}

The ASER for square QAM, QPSK and coherent detection of differentially encoded PSK with SWC is given by $P_{S}=\frac{2 a}{\pi} \int_{0}^{\pi / 2} \phi_{z}\left(b \csc ^{2} \theta\right) d \theta-\frac{4 c}{\pi} \int_{0}^{\pi / 4} \phi_{z}\left(b \csc ^{2} \theta\right) d \theta$. By solving the quadratic problem $\operatorname{cerfc}^{2}(\sqrt{b \xi})-a \operatorname{erfc}(\sqrt{b \xi})+P_{s}^{(.)}=0$ for $\xi$ (i.e., Eq. (15)), we obtain a closed-form expression for the optimal switching threshold,

$$
\xi^{*}=\frac{1}{b}\left\{\operatorname{erfcinv}\left[\frac{a-\sqrt{a^{2}-4 c P_{s}^{(x)}}}{2 c}\right]\right\}^{2}
$$

For instance, the optimal switching threshold for the QPSK or the 4-QAM modulation scheme is given by

$$
\xi^{*}=\left(\operatorname{erfcinv}\left(2\left[1-\sqrt{1-P_{s}^{(x)}}\right]\right)\right)^{2}
$$

where $P_{s}^{(x)}=\frac{2}{\pi} \int_{0}^{\pi / 2} \phi_{x}\left(\csc ^{2} \theta\right) d \theta-\frac{1}{\pi} \int_{0}^{\pi / 4} \phi_{x}\left(\csc ^{2} \theta\right) d \theta$.

\section{Correlated Fading and Identical Diversity Branches}

If the assumption of branch independence is slightly relaxed, then we may only get the optimal $\xi$ in a closed-form for the exponential form of $P_{s}(\varepsilon \mid \gamma)$ in correlated Rayleigh and/or correlated Nakagami-m fading channels. Following our treatment for the independent signal fading, the optimal switching threshold for the differentially coherent or the noncoherent binary modulation format is obtained by solving $\frac{\partial}{\partial \xi} \phi_{z}(b)=0$ for $\xi$ :

$$
\phi_{r}(b, \xi)-f_{x}(\xi) \exp (-b \xi)=0
$$

Substituting the marginal MGF for the correlated Nakagami-m fading channel with identical mean received signal strength (see Table 1) in (20), we get

$$
\xi^{*}=m\left[\frac{m+b \Omega(1-\rho)}{b(1-\rho)(m+b \Omega)}\right] \ln \left(1+\frac{b \Omega(1-\rho)}{m}\right)
$$

which is a generalization of Eq. (24) in [6] for the differentially coherent binary signalling scheme and for arbitrary $m$ values. By setting the power correlation coefficient $\rho=0$, (21). reduces to (16). For other modulation formats listed in Table 1 of [9], $\xi^{*}$ is given by the unique real positive root of (22), which will be determined numerically:

$$
\sum_{k} \int_{0}^{n_{k}} a_{k}(\theta) \phi_{x}\left(b_{k}(\theta), \xi\right) d \theta-f_{x}(\xi) P_{S}(\varepsilon \mid \xi)
$$

As an illustrative example, let us the characterize the SWC performance (with optimum switching threshold) for different M-ary signalling constellations in Rayleigh and Rician fading channels. For the Rician fading channel, the CDF of signal power (for a single branch) is given by

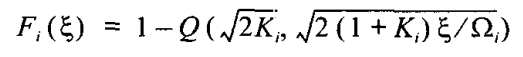

and

$$
\begin{gathered}
\int_{\xi}^{\infty} \exp (-s u) f_{i}(u) d u=\frac{1+K_{i}}{s \Omega_{i}+K_{i}+1} \exp \left(\frac{-s K_{i} \Omega_{i}}{s \Omega_{i}+K_{i}+1}\right) \\
\times Q\left(\sqrt{\frac{2 K_{i}\left(K_{i}+1\right)}{s \Omega_{i}+K_{i}+1}}, \sqrt{\frac{2\left(s \Omega_{i}+K_{i}+1\right) \xi}{\Omega_{i}}}\right)
\end{gathered}
$$

where $Q(\sqrt{2 a}, \sqrt{2 b})=\int_{b}^{\infty} \exp (-t-a) I_{0}(2 \sqrt{a}) d t$ is the Marcum-Q function and $i \in\{x, y\}$. If the received signal envelopes from the two antennas are assumed to be independent and identically distributed, then $\Omega_{i}=\Omega$ and $K_{i}=K$. Substituting (23) and (24) into (10), we get a closed-form expression for $\phi_{z}($.$) , i.e.,$ 


$$
\begin{gathered}
\phi_{=}(s)=\frac{1+K}{s \Omega+K+1} \exp \left(\frac{-s K \Omega}{s \Omega+K+1}\right) \\
\times\left[1+Q\left(\sqrt{\frac{2 K(K+1)}{s \Omega+K+1}}, \sqrt{\frac{2(s \Omega+K+1) \xi^{*}}{\Omega}}\right)\right. \\
\left.-Q\left(\sqrt{2 K}, \sqrt{\frac{2(1+K) \xi^{*}}{\Omega}}\right)\right]
\end{gathered}
$$

and the optimal threshold $\xi^{*}$ is calculated using (18). For Rayleigh fading, (25) reduces to

$\phi_{=}(s)=\frac{1}{s \Omega+1}\left[1-\exp \left(-\xi^{*} / \Omega\right)+\exp \left(-(s \Omega+1) \xi^{*} / \Omega\right)\right](26)$ since $Q(0, \beta)=\exp \left(-\beta^{2} / 2\right)$.

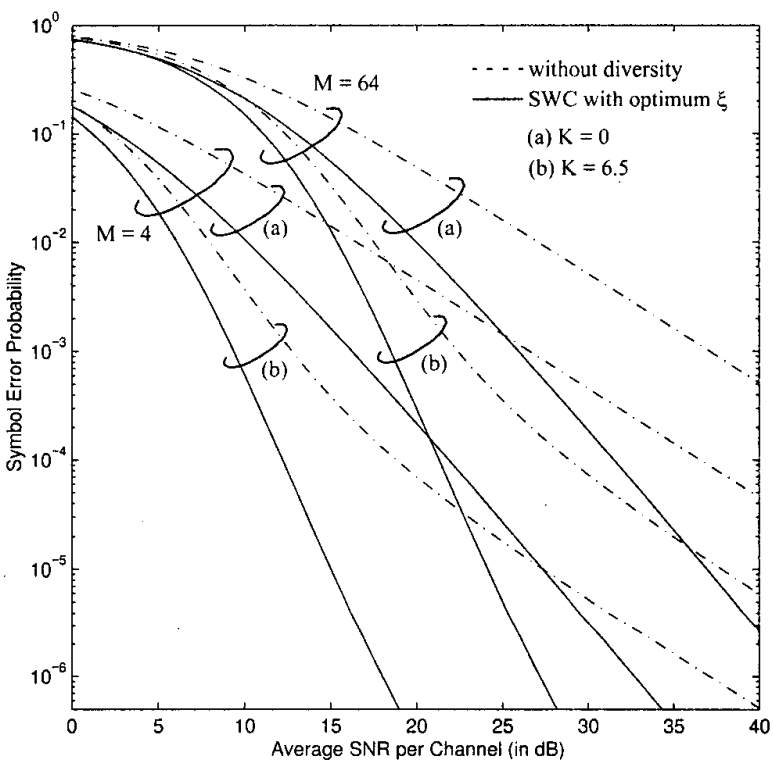

Fig 3. Performance of MQAM with SWC in Rayleigh and Rician fading channels.

From Fig. 3, it is apparent that the diversity reception is a simple yet powerful technique for mitigating the effect of deep fades experienced in wireless channels. For instance, the dual-branch SWC system can reduce the penalty in the required SNR to achieve an error rate of $P_{S}=10^{-3}$ for the 64-QAM by approximately $11 \mathrm{~dB}$ in a Rayleigh fading channel with respect to the no diversity case. The diversity advantage is greater for a larger alphabet size and in a poorer channel condition (i.e., as $K \rightarrow 0$ ), as anticipated.

\section{CONCLUSIONS}

This paper presents a concise, unified approach for evaluating the switched diversity performance for a wide range of modulation formats and fading environments. Recent studies [8-13] have shown that the MGF enables the rapid computation of the ABER and ASER. Therefore, this paper derives the MGF of the SWC output directly. As well, the derivative of the MGF follows at once, which is used to determine the optimal (in the minimum error rate sense) switching threshold. Commonly used MGFs are tabulated for the use with our generic expressions. Results of [6]-[8] are presented as special cases of our analysis. Closed-form expressions for the optimal switching threshold are derived for three generic forms of conditional SER probability by assuming identical fading statistics across the statistically independent diversity branches. When the effect of branch correlation is taken into account, a closed-form formula for the optimal switching threshold is only available for the exponential form of conditional error probability in Rayleigh and Nakagami-m fading channels with equal mean received signal strength. A comprehensive study of switched diversity systems with arbitrary fading parameters can be found in [14].

\section{REFERENCES}

[1] A. J. Rustako Jr., Y. S. Yeh and R. R. Murray, "Performance of Feedback and Switch Space Diversity $900 \mathrm{MHz}$ FM Mobile Radio Systems with Rayleigh Fading," IEEE Trans. Communications, Vol. 21, pp. 1257-1268, Nov. 1993.

[2] W. E. Shortall, "A Switched Diversity Receiving System for Mobile Radio," IEEE Trans. Communications, Vol. 21, pp. 1269-1275, Nov. 1973.

[3] F. Adachi, T. Hattori, K. Hirade and T. Kamata, "A Periodic Switching Diversity Technique for a Digital FM Land Mobile Radio," IEEE Trans. Vehicular Technology, Vol. 27, pp. 211-219, Nov. 1978.

[4] M. A. Blanco and K. J. Zdunek, "Performance and Optimization of Switched Diversity Systems for Detection of Signals with Rayleigh Fading," IEEE Trans. Communications, Vol. 27, pp. 1887-1895, Dec. 1979.

[5] M. Blanco, "Diversity Receiver Performance in Nakagami Fading," Proc. 1983 IEEE Southeastern Conference, Orlando, pp. 529-532.

[6] A. Abu-Dayya and N. C. Beaulieu, "Analysis of Switched Diversity System on Generalized-Fading Channels," IEEE Trans. Communications, Vol. 42, pp. 2959-2966, Nov. 1994.

[7] A. Abu-Dayya and N.C. Beaulieu, "Switched Diversity on Microcellular Rician Channels," IEEE Trans. Vehicular Technology, Vol. 43, pp. 970-976, Nov. 1994.

[8] M. K. Simon and M. -S. Alouini, Tutorial Notes TU 08: A Unified Approach to the Error Probability Analysis of Digital Communication over Generalized Fading Channels, IEEE Global Telecommunications Conference, Sydney, 8-12 Nov. 1998.

[9] A. Annamalai, C. Tellambura and V. K. Bhargava, "Unified Analysis of Equal-Gain Diversity on Rician and Nakagami Fading Channels," Proc. IEEE WCNC'99.

[10] C. Tellambura, A. J. Mueller and V. K. Bhargava, "Analysis of M-ary Phase-Shift-Keying with Diversity Reception for Land-Mobile Satellite Channels," IEEE Trans. Vehicular Technology, Vol. 46, pp. 910-922, November 1997.

[11] C. Tellambura and V. K. Bhargava, "Unified Error Analysis of DQPSK in Fading Channels," IEE Electronics Letters, Vol. 30, No. 25, pp. 2110-2111, Dec. 1994.

[12] A. Annamalai, C. Tellambura and V. K. Bhargava, "Unified Analysis of MPSK and MDPSK with Diversity Reception in Different Fading Environments," IEE Electronics Letters, Vol. 34, No. 16, 6th August 1998, 1564-1565.

[13] M. Simon and M. Alouini, "A Unified Approach to Performance Analysis of Digital Communication over Generalized Fading Channels," Proc. IEEE, Vol. 86, September 1998, pp. 1860-1877.

[14] C. Tellambura, A. Annamalai and V. K. Bhargava, "Unified Analysis of Switched Diversity Systems in Independent and Correlated Fading Channels," submitted to IEEE Trans. Communications. 